\title{
Variations on a Theme in Paper Folding
}

\author{
Burkard Polster
}

1. INTRODUCTION. Starting in 1971 , Jean Pedersen published a number of articles in which she introduced and investigated an ingenious paper-folding construction for approximating certain rational angles and regular star polygons. Later on, she and Peter Hilton wrote a series of joint articles in which they generalized this construction, thereby allowing one to approximate any rational angle and any regular star polygon. Furthermore, they did an in-depth investigation of the number-theoretical ideas that their construction suggested. See [1], [2], [4], and [5] for excellent surveys of their work on this topic.

When first encountered, the Hilton-Pedersen construction produces a magical "AHA!" effect similar to the one produced by a Möbius strip cut in half. Just like the Möbius strip trick, it uses only a simple strip of paper. It can be put to good use in the classroom, as a mathematical magic trick to impress nonmathematical friends and, last but not least, as a source of some very pretty nontrivial mathematics. Anyway, once the reader has understood what it is all about, he or she will never forget it.

In this article, we summarize the construction in a way that enables us to describe other related paper folding constructions as alternative geometrical front-ends to the sound mathematical base created by Hilton and Pedersen. One such construction is Fujimoto's method for approximating rational subdivisions of arbitrary angles and line segments as described in [7].

To really appreciate the beauty of the constructions presented in what follows, the reader is strongly encouraged to try them out with real paper.

2. THE HILTON-PEDERSEN CONSTRUCTION. The Hilton-Pedersen construction splits into two parts. First, an angle-folding algorithm, which is the part of the construction that has the different geometrical front-ends that this paper is all about. Second, the so-called FAT-algorithm for turning the output of the angle-folding algorithm into regular star polygons. Our focus is on the angle-folding algorithm, which we present in such a way that all the geometrical front-ends attach to it seamlessly. For more details about the results mentioned in this section, we refer the reader to any of the survey articles listed earlier.

The angle-folding algorithm. We start with an acute rational angle (an angle that is a rational multiple of $\pi) \alpha_{0}=a_{0} \pi / n$, where $a_{0}$ and $n$ are relatively prime positive integers and $n$ is odd. ${ }^{1}$ Using a strip of paper whose two long edges are parallel, we fold this angle as in Figure 1. For the moment, we stick with virtual paper and don't

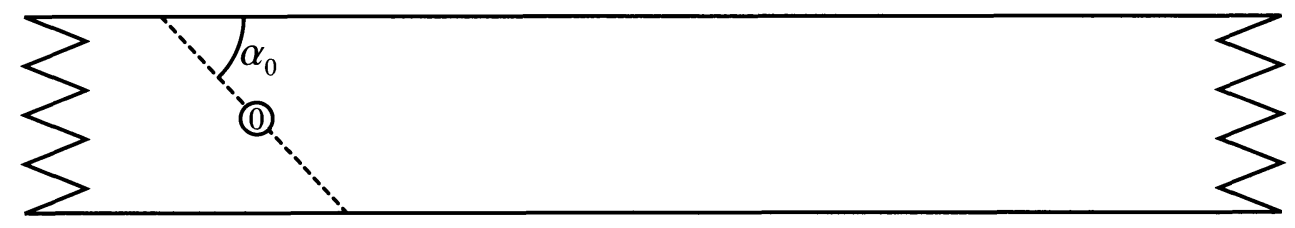

Figure 1. Angle $\alpha_{0}=a_{0} \pi / n$ folded into a strip of paper with parallel edges.

${ }^{1}$ We explain later why this is not a serious restriction. 
worry about how one would actually go about folding $\alpha_{0}$ into a real strip of paper. Note that in the diagrams, we label creases in the paper in the order in which we produce them.

Depending on whether $a_{0}$ is an even or an odd number, we proceed in one of two ways, which we call a fold and a switchfold, respectively.

Fold ( $a_{0}$ even): In this case, we bisect the angle $\alpha_{0}$ by folding the original crease onto the edge that borders this angle (Figure 2). If we set $a_{1}=a_{0} / 2$, then the resulting angle is $\alpha_{1}=a_{1} \pi / n$.

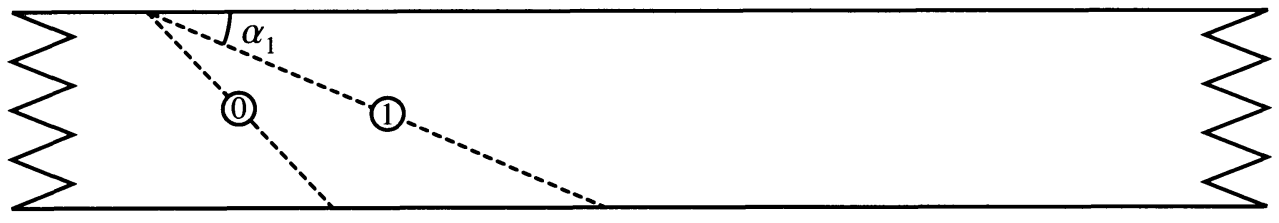

Figure 2. If $a_{0}$ is even, perform a fold by bisecting $\alpha_{0}$.

Switchfold ( $a_{0}$ odd): In this case, we bisect the angle $\left(n-a_{0}\right) \pi / n$ on the other side of the original crease (Figure 3). If we set $a_{1}=\left(n-a_{0}\right) / 2$, then the new angle is $\alpha_{1}=a_{1} \pi / n$. Note that since $n$ is odd, $a_{1}$ is an integer.

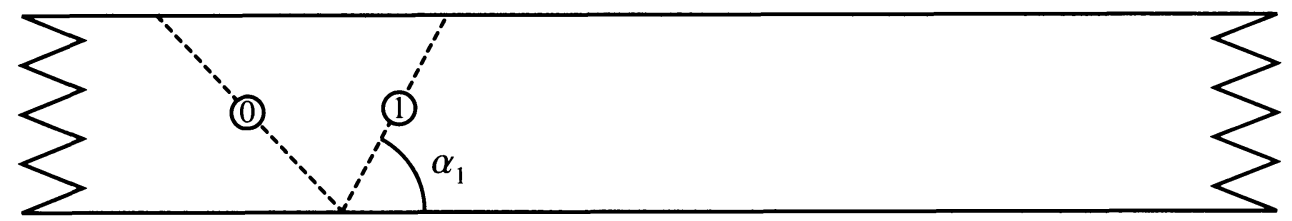

Figure 3. If $a_{0}$ is odd, perform a switchfold; that is, "switch" to the angle "opposite" $\alpha_{0}$ and bisect it.

In both cases, the resulting angle $\alpha_{1}=a_{1} \pi / n$ is acute, and $a_{1}$ and $n$ are relatively prime. We iterate this procedure to construct a sequence of angles $\alpha_{0}, \alpha_{1}, \alpha_{2}, \ldots$, as well as the paper-folding sequence corresponding to $\alpha_{0}$ that consists of folds and switchfolds. It is easy to prove that the value $\alpha_{0}$ appears repeatedly in the angle sequence. If we let $m$ denote the smallest positive integer such that $\alpha_{0}=\alpha_{m}$, then it is also clear that the sequence of angles constructed is cyclic of period $m$.

For example, let $\alpha_{0}=\pi / 3$. Then, all the angles in the first sequence are $\pi / 3$, and the paper-folding sequence corresponding to $\pi / 3$ is

$$
\text { switchfold - switchfold - switchfold } \cdots .
$$

The corresponding folded strip of paper is shown in Figure 4.

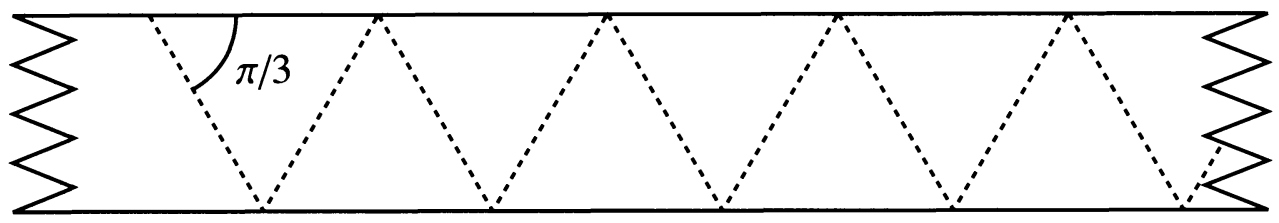

Figure 4. The crease pattern corresponding to the angle $\pi / 3$. 
For later use, we list the paper-folding sequence of $\pi / 5$, which is

$$
\text { switchfold - fold - switchfold - fold - switchfold - fold } \cdots,
$$

and that of $\pi / 7$, which is

$$
\text { switchfold - switchfold - fold - switchfold - switchfold-fold } \cdots .
$$

Approximating angles. Using a new strip of paper-this also works with real paperwe fold an arbitrary approximation $\beta_{0}$ to the angle $\alpha_{0}$, and continue folding according to the paper-folding sequence generated by $\alpha_{0}$. It turns out that as $i$ goes to infinity the angle $\beta_{i m}$ constructed by such a method will converge rapidly to $\alpha_{0}$. (Here $m$ is the period of the angle sequence corresponding to $\alpha_{0}$.) This means that, in this way, we can construct arbitrarily good approximations to $\alpha_{0}$ with very little effort. For example, if we start with an approximation $\beta_{0}$ to $\pi / 3$ and apply the paper-folding sequence of $\pi / 3$ to it, then we are very quickly led to a sequence of creases that is indistinguishable from the one that we constructed starting with $\pi / 3$ itself (Figure 5).

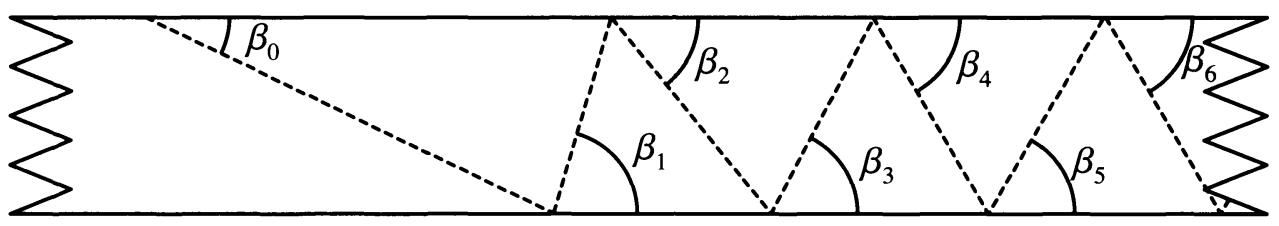

Figure 5. The crease pattern corresponding to an approximation to $\pi / 3$.

It is a routine exercise to extend the angle-folding algorithm to a method for approximating any rational angle. As a first important step towards realizing such a method, just note that once we have found a good approximation to $a_{0} \pi / n$, a rational angle with odd "denominator," we can successively fold this angle in half $r$ times to construct a good approximation to the rational angle $a_{0} \pi /\left(2^{r} n\right)$, which has an even denominator.

Approximating regular polygons and star polygons. Let $a \pi / n$ again be one of the possible input angles to the angle-folding algorithm. We fold an approximation to this angle into a long strip of paper and apply the corresponding folding sequence to this approximation "lots of times." From what we said before, it is clear that from some point on the resulting crease pattern will be indistinguishable from the crease pattern we arrive at by starting with the angle $a \pi / n$ itself. For the following to work, it is important that we produce all creases in the same manner, that is, when we look at one side of the creased strip of paper either all creases look like valleys or all look like ridges.

The FAT-algorithm of Hilton and Pedersen is a systematic way to "Fold And Twist" along some of the creases of the crease pattern to arrive at a regular (star) $\{n / a\}$-gon; i.e., a connected sequence of edges that visits every $a$ th vertex of a regular $n$-gon. Figure 6 shows two regular star polygons produced in this manner.

Start by selecting $2 n$ equally spaced pairs of creases such that the two creases in a given pair intersect at the top edge of the strip and such that both the angle between the two creases and the angle between the top edge and the top crease are $a \pi / n$. The reader can convince himself or herself that this is always possible. For example, Figure 7a shows part of a crease pattern that corresponds to both $\pi / 7$ and $2 \pi / 7$ (since "folding" $2 \pi / 7$ gives $\pi / 7$ ). Figure $7 b$ shows how to select pairs of creases in each case. 

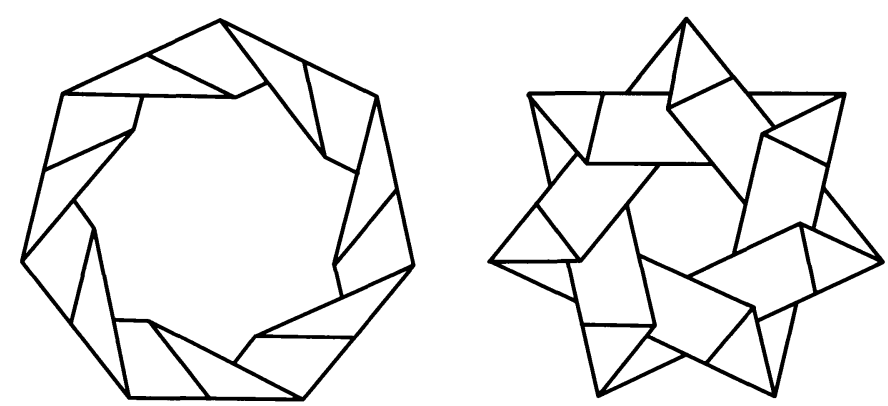

Figure 6. A regular 7-gon (= regular star $\{7 / 1\}$-gon) and a regular star $\{7 / 2\}$-gon produced with the FATalgorithm.

(a)
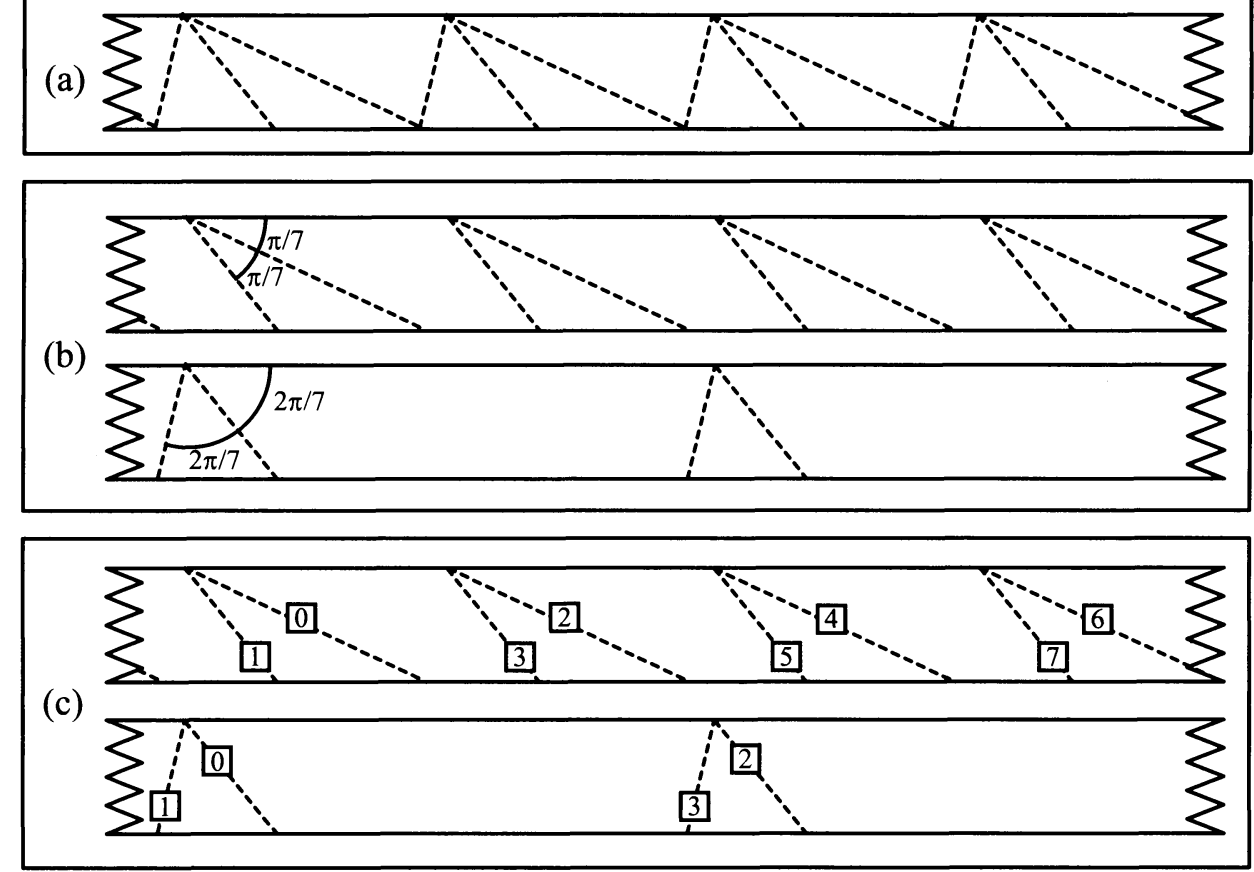

Figure 7. (a) The crease pattern corresponding both to an approximation to $\pi / 7$ and to $2 \pi / 7$; (b) two selections of pairs of creases; (c) orders in which to fold and twist along the selected creases to produce a regular 7 -gon and a regular $\{7 / 2\}$-gon, respectively.

Following these choices, we number the selected creases as indicated in Figure 7c. Note that in the second selection we choose only every second possible pair of creases in order to allow for enough room to "weave in" the resulting strip as in the right diagram in Figure 6.

We now return to the general case. We fold the strip flat along crease 0 and keep it folded. This aligns crease 1 with one of the edges. Then we fold the strip flat along crease 1 over this edge and, again, keep it folded. This produces the first corner of the $\{n / a\}$-gon we are after and also results in a full twist of the strip of paper. We fold crease 2 followed by crease 3 to produce the second corner, and so forth. Mainly for aesthetic reasons, we also weave in the growing star polygon as we fold and twist away. 
We note in passing that the extension of the angle-folding algorithm to a method for approximating any rational angle mentioned earlier gives rise in a natural manner to an extension of the FAT-algorithm that approximates any regular $\{n / a\}$-gon. However, if either $n$ or $a$ is even, the situation can become more complicated, and may involve some secondary folds. It is also possible, and a lot of fun, to use the creased strips of paper constructed above to plait regular polyhedra (see, for example, [3]).

3. VARIATION 1: FOLDING IN CIRCLES. For this variation simply replace the strips of paper that we have used so far with circular pieces of paper. Figure 8 illustrates what we have in mind; namely, how exactly folding an angle and performing a fold and a switchfold translate into this alternative setting.
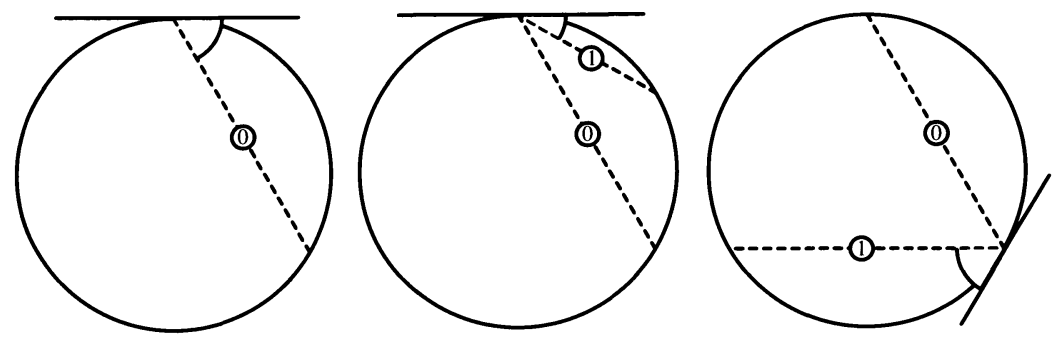

Figure 8. Fold the angle $a \pi / n$ as in the left-hand diagram. If $a$ is even, fold as in the middle diagram. Otherwise, switchfold as in the right-hand diagram.

As before, we start by folding an acute angle $a \pi / n$, with $n$ odd and relatively prime to $a$. It is easy to see that, even in the circle setting, the angle-folding algorithm applied to this angle yields exactly the same paper-folding sequence as in the strip setting.

In the circle setting, constructing regular $\{n / a\}$-gons is straightforward. Just fold the initial angle $a \pi / n$ and then apply the corresponding paper-folding sequence. Figure 9 shows what happens in the case of the angles $\pi / 3, \pi / 5$, and $\pi / 7$ (in the last case we only draw the first four creases). Another interesting fact about this construction is that all the creases that are produced are diagonals of the target regular star $\{n / a\}$-gon.
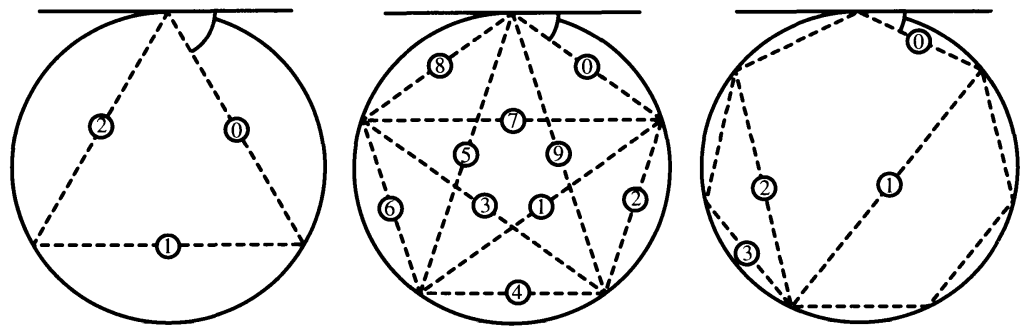

Figure 9. Constructing an equilateral triangle, a regular 5-gon, and a regular 7-gon using the folding sequences corresponding to the angles $\pi / 3, \pi / 5$, and $\pi / 7$, respectively, on a circular piece of paper.

Of course, if we start with an arbitrary angle and apply the paper-folding sequence of $a \pi / n$ to it, we will be constructing better and better approximations to this angle as we continue folding. This implies that we will also be approximating a regular star $\{n / a\}$-gon. 


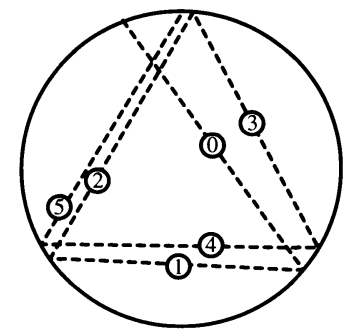

Figure 10. Approximating an equilateral triangle using the paper-folding sequence corresponding to $\pi / 3$.

Using the example in Figure 10, we indicate how this last statement can be made precise. Here, an equilateral triangle is being approximated using the paper-folding sequence corresponding to $\pi / 3$. In this special case, we can consider the last three creases constructed at any stage as an approximation to an equilateral triangle. This approximation will improve rapidly as we keep folding away.

4. VARIATION 2: DISSECTING ANGLES INTO EQUAL PARTS. In the following, we identify a construction by Fujimoto [7] as a variation of the angle-folding algorithm. In this variation, we change two things. First, we replace the strip of paper with a wedge of paper whose determining angle is $\Delta$ (Figure 11). Second, we replace $\pi$ in all the angles with $\Delta$. So, for example, we start with the angle $a \Delta / n$ instead of $a \pi / n$.

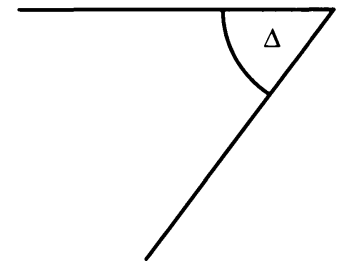

Figure 11. A wedge of paper.

Figure 12 illustrates how folding an angle and performing a fold and a switchfold translate into the wedge context. As usual, we start by folding an angle $a \Delta / n$, where $n$ is odd, relatively prime to $a$, and greater than $a$. As in the circular situation, it is straightforward to see that in the wedge setting the angle-folding algorithm applied to the angle $a \Delta / n$ yields exactly the same paper-folding sequence as the algorithm applied to the angle $a \pi / n$ in the strip framework.
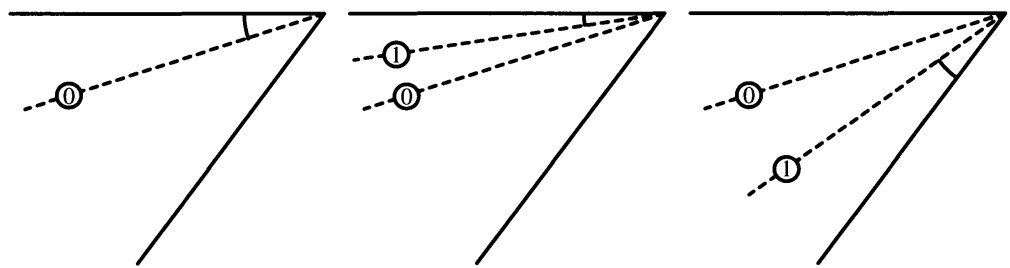

Figure 12. Fold the angle $a \Delta / n$ as in the left-hand diagram. If $a$ is even, fold as in the middle diagram. Otherwise, switchfold as in the right-hand diagram. 

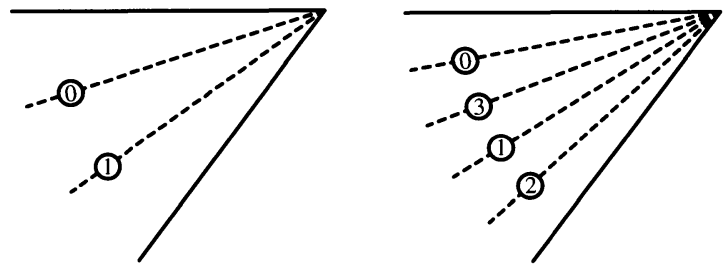

Figure 13. Sequences of creases corresponding to the angles $\Delta / 3$ and $\Delta / 5$.

Figure 13 shows the creases that are produced for the starting angles $\Delta / 3$ and $\Delta / 5$. Note that these are all the creases one will ever get no matter how long one keeps folding. For example, for $\Delta / 3$ one will fold crease 0 on one step, then crease 1 , then crease 0 again, and so on.

Of course, if we apply the paper-folding sequence corresponding to $a \Delta / n$ to any positive angle smaller than $\Delta$, we end up approximating $a \Delta / n$. In the simplest case where $a=1$ and $n=3$, we achieve the "impossible" by trisecting the angle $\Delta$. Well, not really, since we are only talking about an approximation, no matter how good and fast it might be. However, we should note at this point that the impossible really is possible with origami in that angles can be trisected and cubes doubled exactly using origami techniques (see [6] for more details).

It is also interesting to consider what happens if we let $\Delta$ take on the extreme values 0 and $2 \pi$. At first glance, the first case gives nothing interesting because the wedge turns into a line. However, we may consider any infinite strip of paper with parallel edges as a wedge with determining angle 0 whose edges meet at infinity (see the left-hand diagram in Figure 14). In this case the creases run parallel to the edges of the strip, and we can use the paper-folding sequence corresponding to $\Delta / n$, derived for positive $\Delta$, to approximate a subdivision of the strip into $n$ horizontal strips of equal height. We note that there are exact methods to achieve the same using origami.

In the case $\Delta=2 \pi$, we may consider the wedge as a circular piece of paper that has been cut along one of its radii (see the right-hand diagram in Figure 14). In this instance, the creases are radii of the circle, and we use the paper-folding sequence corresponding to $\Delta / n$ to approximate the $n$ "spokes" of a regular $n$-gon inscribed in the circle such that one of the spokes is the cut in the circle.
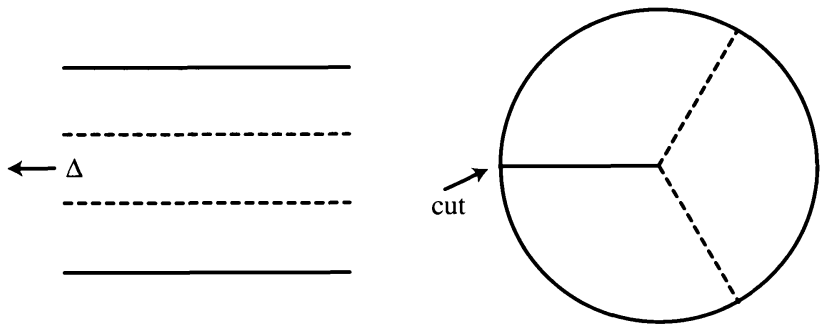

Figure 14. Sequences of creases corresponding to the angle $\Delta / 3$ in the extreme cases $\Delta=0$ and $\Delta=2 \pi$, respectively.

5. VARIATION 3: MORE DISSECTING OF ANGLES. The following variation is due to Pedersen (see, for example, [8]). In it we once more replace the strip of paper with a wedge of angle $\Delta$ and replace the angle $\pi$ in the definitions of folding and switchfolding with the angle $\pi-\Delta$. However, this time the angle $\Delta$ has to be smaller 

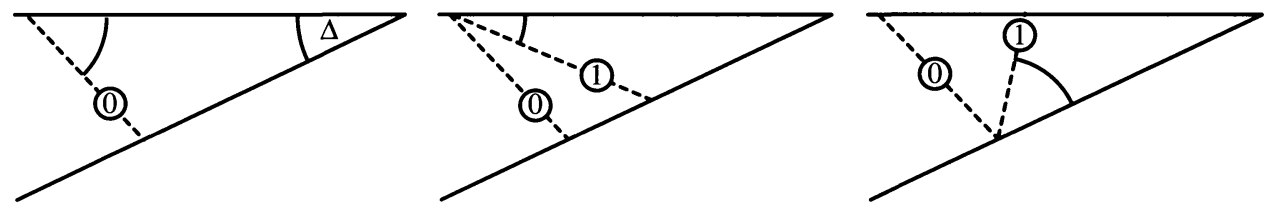

Figure 15. Fold the angle $a(\pi-\Delta) / n$ as in the left-hand diagram. If $a$ is even, fold as in the middle diagram. Otherwise, switchfold as in the right-hand diagram.

than $\pi$, and the way we fold is the same as in the case of a strip of paper (see Figure 15 and compare the three diagrams in this figure with those in Figures 1, 2, and 3).

Note that since the starting angle is $a(\pi-\Delta) / n$ with $a<n$, we can be sure that the first and all subsequent creases actually intersect both edges of the wedge. We can make the usual remark that nothing of substance changes in the algorithm. In particular, if we apply the paper-folding sequence of $a(\pi-\Delta) / n$ to any possible positive angle, we end up approximating $a(\pi-\Delta) / n$ (see Figure 16 for an example corresponding to the initial angle $\Delta / 3$ ).

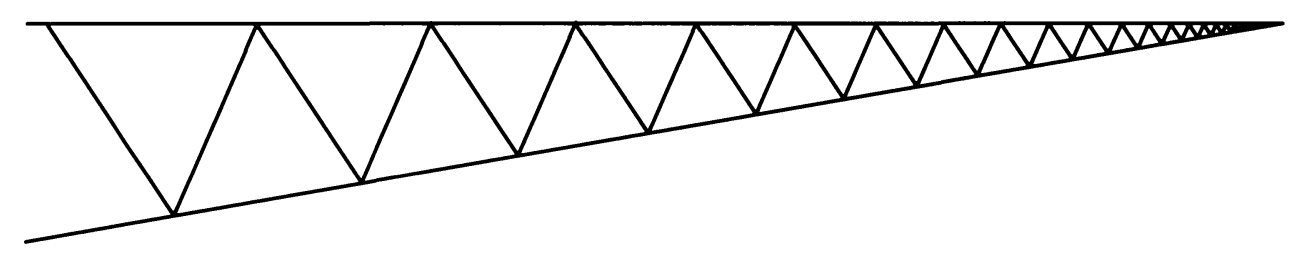

Figure 16. The crease pattern corresponding to the angle $(\pi-\Delta) / 3$ for small $\Delta$.

We can use the creased strips produced by this method to fold self-similar spirals. Figure 17 offers an example of this.

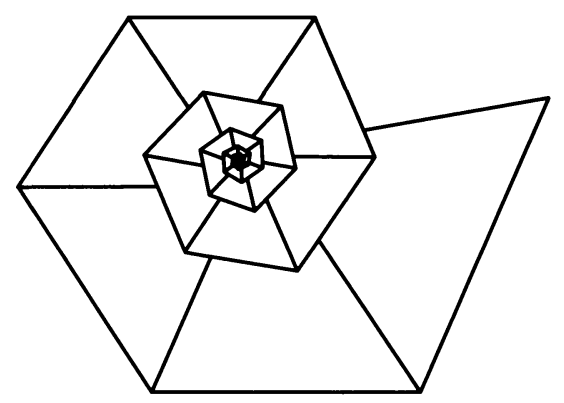

Figure 17. Folding the creased wedge in the previous figure into a spiral.

Observe that if we let $\Delta=0$, in the same sense as in the previous variation, this final variation turns into the strip setting that we started with.

ACKNOWLEDGMENTS. I would like to thank Professors Jean Pedersen and Peter Hilton for their help in finetuning this article. 
$\rightarrow$ P. Hilton and J. Pedersen, Approximating any regular polygon by folding paper, Math. Mag. 56 (1983) 141-155.

2. - Folding regular star polygons and number theory, Math. Intelligencer 7 (1985) 15-26.

3. — Build Your Own Polyhedra, Dale Seymour, Palo Alto, CA, 1999.

4. P. Hilton, D. Holton, and J. Pedersen, Mathematical Reflections: In a Room with Many Mirrors, SpringerVerlag, New York, 1998.

5. — Mathematical Vistas: From a Room with Many Windows, Springer-Verlag, New York, 2002.

$\rightarrow$ T. Hull, A note on "impossible" paper folding, this MONTHLY 103 (1996) 240-241.

7. H. Huzita and S. Fujimoto, Fujimoto successive method to obtain odd-number section of a segment or an angle by folding operations, in Origami Science and Art, Proceedings of the Second International Meeting of Origami Science and Scientific Origami (Otsu, Japan, November 29-December 2, 1994), Koryo Miura, ed., published by the organizing comittee with the support of the Seian University of Art and Design, 1997, pp. 1-13.

8. J. Pedersen, Asymptotic euclidean constructions without euclidean tools, Fibonacci Quart. 9 (1971) 199216.

BURKARD POLSTER is well known in and around his home town Melbourne, Australia, as a mathematical juggler, bubble master, origami expert, and shoelace charmer. When he is not busy doing fun mathematics, he has fun doing research in finite and topological geometry as a senior research fellow in the School of Mathematical Sciences at Monash University.

School of Mathematical Sciences, Monash University, 3800 Victoria, AUSTRALIA

Burkard.Polster@sci.monash.edu.au

http://www.maths.monash.edu.au bpolster 